\title{
SOME ASYMPTOTIC FORMULAS FOR WIENER INTEGRALS $\left({ }^{1}\right)$
}

\author{
BY \\ M. SCHILDER
}

1. Introduction. In this paper asymptotic formulas for Wiener integrals similar to the Laplace asymptotic formulas for integrals in one dimension will be proved [5], and some applications to analysis of these formulas will be given.

Let $C[0, T]$ be the space of continuous functions defined on the interval $[0, T]$ which vanish at zero, and let $\|z\|$ be the sup norm of a function $z$. A functional $H(z)$ will be said to have a proper maximum at a point $x$ if $H(z)<H(x)$ for $z \neq x$.

Let $C^{*}[0, T] \subset C[0, T]$ be the subset of functions on $[0, T]$ which are absolutely continuous and whose derivatives are in $L^{2}[0, T]$.

Suppose $F(z)$ is a functional on $C[0, T]$ such that $F(z)-\frac{1}{2} \int_{0}^{T}\left(z^{\prime}(\tau)\right)^{2} d \tau$ has a proper maximum on $C^{*}[0, T]$. Call this maximizing function $x(\tau)$. With further restrictions on $F(z)\left({ }^{2}\right)$ but not supposing $F(z)$ to have any Frechet derivatives, and for a large class of functionals $G(z)$, it will be $\operatorname{shown}\left({ }^{2}\right)$ that

$$
\lim _{\lambda \rightarrow 0} \frac{E_{z}^{w}\left\{G(\lambda z) \exp \left(\lambda^{-2} F(\lambda z)\right)\right\}}{E_{z}^{w}\left\{\exp \left(\lambda^{-2} F(\lambda z)\right)\right\}}=G(x)
$$

where $E_{z}^{w}\{\}$ denotes integration with respect to Wiener measure [8].

In the case that $F(z)$ and $G(z)$ have Frechet derivatives in a neighborhood of $x$, it will be shown that

$$
\exp \left(-b \lambda^{-2}\right) E_{z}^{w}\left\{G(\lambda z) \exp \left(\lambda^{-2} F(\lambda z)\right)\right\}=\Gamma_{0}+\Gamma_{1} \lambda+\cdots+\Gamma_{R} \lambda^{K}+O\left(\lambda^{K+1}\right)
$$

where $b=F(x)-\frac{1}{2} \int_{0}^{T}\left[x^{\prime}(\tau)\right]^{2} d \tau$ and the $\Gamma_{\mathfrak{t}}$ are Wiener integrals not depending on $\lambda$.

In $\S 2$, the exact conditions under which (1.1) and (1.2) hold will be given. In $\S 3$, several lemmas necessary for the proof of (1.1) and (1.2) will be given, and in $\S 4$, the proof of (1.1) and (1.2) will be completed. In $\$ 5$, some applications of (1.1) are given.

Formulas (1.1) and (1.2) first arose in connection with a problem in nonlinear partial differential equations.

Received by the editors June 1, 1965 and, in revised form, December 28, 1965.

(1) Part of this paper was submitted as a doctoral thesis at the Courant Institute of Mathematical Sciences, New York University under Professor M. Dansker.

$\left({ }^{2}\right)$ See $\$ 2$. 
2. Some asymptotic formulas for Wiener integrals. The main results of this paper are:

THEOREM $\mathrm{A}\left({ }^{3}\right)$. Suppose $F(z)$ and $G(z)$ are two real valued functionals defined on $C[0, T]$, and suppose that the functional $F(z)-\frac{1}{2} \int_{0}^{T}\left[z^{\prime}(\tau)\right]^{2} d \tau$ has a proper maximum at $x$ over $C^{*}[0, T]$. If $F(z)$ and $G(z)$ satisfy conditions $1-5$ below, then

$$
\lim _{\lambda \rightarrow 0} \frac{E_{z}^{w}\left\{G(\lambda z) \exp \left(\lambda^{-2} F(\lambda z)\right)\right\}}{E_{z}^{w}\left\{\exp \left(\lambda^{-2} F(\lambda z)\right)\right\}}=G(x)
$$

Conditions 1-5 are:

1. $G(z)$ and $F(z)$ are Wiener measurable.

2. $|G(z)| \leqq K_{1} \exp \left(K_{2}\|z\|^{2}\right)$ almost everywhere with respect to Wiener measure where $K_{1}$ and $K_{2}$ are any positive real numbers.

3. $F(z) \leqq L_{1}+L_{2}\|z\|^{2}$ almost everywhere with respect to Wiener measure where $L_{1}$ is any positive real number but $L_{2}<T / 4$.

4. $G(z)$ is continuous at $x$ in the sup norm.

5. $F(z)$ is continuous for

$$
\|z\|<\left|\left(b-L_{1}+1\right) /\left(2 L_{2}-1 / 2 T\right)\right|^{1 / 2},
$$

and upper semicontinuous almost everywhere in the sup norm.

For convenience the following notation is introduced. If

$$
\frac{\partial^{K} F(x+\eta)}{\partial\left(x\left(s_{1}\right)+\eta\left(s_{1}\right)\right) \cdots \partial\left(x\left(s_{K}\right)+\eta\left(s_{K}\right)\right)}
$$

is the $K$ th Frechet derivative of the functional $F(z)$ at the point $x+\eta(\eta \in C[0, T])$, then the expression

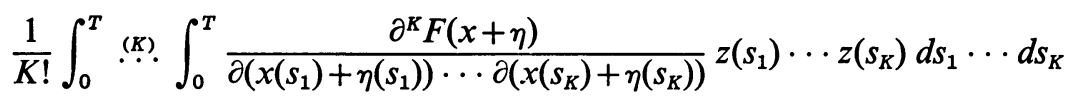

will be denoted by $f_{K}(\eta) z^{K}$.

THEOREM B. Suppose $F(z)$ and $G(z)$ are defined on $C[0, T]$ and satisfy the hypothesis of Theorem A, and suppose also, that

1. $F(z)$ has two continuous Frechet derivatives in a uniform neighborhood of the maximizing function $x$.

2. $E_{z}^{w}\left\{\exp \left((1+\varepsilon) f_{2}(\eta) z^{2}\right)\right\}$ is uniformly bounded for $\eta$ in a $\delta$ uniform neighborhood of 0 , for some $\varepsilon>0$.

$\left({ }^{3}\right)$ See definitions in the Introduction. 
3. $x^{\prime}(\tau)$ is of bounded variation. Then

$$
\lim _{\lambda \rightarrow 0} \exp \left(-b \lambda^{-2}\right) E_{z}^{w}\left\{G(\lambda z) \exp \left(\lambda^{-2} F(\lambda z)\right)\right\}=G(x) E_{z}^{w}\left\{\exp \left(f_{2}(0) z^{2}\right)\right\}
$$

where $b=F(x)-\frac{1}{2} \int_{0}^{T}\left[x^{\prime}(t)\right]^{2} d t$.

THEOREM C. Suppose $F(z)$ and $G(z)$ are defined on $C[0, T]$ and satisfy the hypothesis of Theorem A, and suppose also that

1. $F(z)$ has $n \geqq 3$ continuous Frechet derivatives in a $\delta$ uniform neighborhood of $x$. It is supposed that $f_{j}(\eta) z^{j}=O\left(\|z\|^{j}\right)$ if $\eta$ is in a $\delta$ neighborhood of zero.

2. $E_{z}^{w}\left\{\exp \left((1+\varepsilon) f_{2}(\eta) z^{2}\right)\right\}$ is uniformly bounded for $\eta$ in a $\delta$ uniform neighborhood of 0 , for some $\varepsilon>0$.

3. $G(z)$ has $n-2$ continuous Frechet derivatives in a $\delta$ uniform neighborhood of $x$.

4. $x^{\prime}(\tau)$ is of bounded variation. Then

$$
\begin{array}{r}
\exp \left(-b \lambda^{-2}\right) E_{z}^{w}\left\{G(\lambda z) \exp \left(\lambda^{-2} F(\lambda z)\right)\right\}=\Gamma_{0}+\Gamma_{1} \lambda+\cdots+\Gamma_{n-3} \lambda^{n-3}+O\left(\lambda^{n-2}\right) \\
\text { as } \lambda \rightarrow 0,
\end{array}
$$

the $\Gamma_{\mathfrak{i}}$ being Wiener integrals depending only on the Frechet derivatives of $F(z)$ and $G(z)$ at $x$.

The assumptions of Theorem A on $F(z)$ and $G(z)$ are strong enough to insure that there exists a $\lambda_{0}$ such that if $\lambda \leqq \lambda_{0}$, then the functional $G(\lambda z) \exp \left(\lambda^{-2} F(\lambda z)\right)$ is Wiener integrable. This is true since

$$
\left|G(\lambda z) \exp \left(\lambda^{-2} F(\lambda z)\right)\right| \leqq K_{1} \exp \left(\lambda^{-2} L_{1}+\left(K_{2} \lambda^{2}+L_{2}\right)\|z\|^{2}\right)
$$

by hypothesis, and from Lemma $1(\S 3)$ it follows that if $\lambda$ is small enough

$$
\begin{aligned}
K_{1} \exp \left(\lambda^{-2} L_{1}\right) & E_{z}^{w}\left\{\exp \left(\left(K_{2} \lambda^{2}+L_{2}\right)\|z\|^{2}\right)\right\} \\
& \leqq 2(2 / \pi T)^{1 / 2} K_{1} \exp \left(\lambda^{-2} L_{1}\right) \int_{0}^{\infty} \exp \left(\left(K_{2} \lambda^{2}+L_{2}\right) u^{2}-u^{2} / 2 T\right) d u<\infty
\end{aligned}
$$

Evidently, the same reasoning may be applied to show that $\exp \left(\lambda^{-2} F(\lambda z)\right)$ is integrable.

For any positive integer $n$, we will denote by $z^{n}$ the vector

$$
z(T / n), z(2 T / n), \ldots, z(T) .
$$

For each $n$-dimensional vector $s^{n}$, we will denote its polygonalization by $s^{n}(\tau)$, i.e.,

$$
s^{n}(\tau)=s_{j}^{n}+\left(\tau-\frac{j T}{n}\right) \frac{n}{T}\left(s_{j+1}^{n}-s_{j}^{n}\right)
$$

for

$$
j T / n \leqq T \leqq(j+1) T / n, \quad j=0,1, \ldots, n-1, \quad s_{0}^{n}=0 .
$$


For each $z \in C[0, T]$, let $z^{n}(\cdot)$ be the polygonalization of the vector $z^{n}$. We will call $z^{n}(\cdot)$ the polygonalization of the function $z$.

If $s^{n}$ is a vector, then $\left\|s^{n}\right\|$ will mean its sup norm.

Define

$$
A(\delta)=\sup _{z \in A} F(z)-\frac{1}{2} \int_{0}^{T}\left[z^{\prime}(\tau)\right]^{2} d \tau-b,
$$

where $A=\left\{z \in C^{*}[0, T] \mid\|z-x\| \geqq \delta\right\}$, and $b$ is defined in the Introduction.

Define $F(z)$ as a function on $R^{n}$ by

$$
F\left(s^{n}\right)=F\left(s^{n}(\cdot)\right) .
$$

3. Lemmas. In this section we state and prove several lemmas necessary for the proof of Theorems A, B, and C.

LeMMA 1. Let $f(u)$ be a nonnegative Lebesgue measurable function on $[0, \infty)$. Then

$$
E_{z}^{w}\{f(\|z\|)\} \leqq 2(2 / \pi T)^{1 / 2} \int_{0}^{\infty} f(u) \exp \left(-u^{2} / 2 T\right) d u
$$

Proof. Let $A$ be any interval contained in $(0, \infty)$. By the symmetry of Wiener measure with respect to reflections across the $x$-axis we have

$$
P\left\{\sup _{0 \leqq \tau \leqq T}|z(\tau)| \in A\right\} \leqq 2 P\left\{\sup _{0 \leqq \tau \leqq T} z(\tau) \in A\right\}
$$

It is well known [5] that

$$
P\left\{\sup _{0 \leqq \tau \leqq T} z(\tau) \in A\right\}=(2 / \pi T)^{1 / 2} \int_{A} \exp \left(-u^{2} / 2 T^{\prime}\right) d u
$$

and hence from a familiar argument

$$
E_{z}^{w}\{f(\|z\|)\} \leqq 2(2 / \pi T)^{1 / 2} \int_{0}^{\infty} f(u) \exp \left(-u^{2} / 2 T\right) d u
$$

LEMMA $\left.2{ }^{4}\right)$. If

$$
\max _{0 \leqq j \leqq n-1}\left[\sup _{j T / n<\tau \leqq(j+1) T / n}|z(\tau)-z(j T / n)|\right] \leqq \delta / 2,
$$

then $\left\|z-z^{n}(\cdot)\right\| \leqq \delta$.

(4) This proof is completely obvious from a picture. 
Proof. Let $\tau_{0}$ be a point where $\left|z(\tau)-z^{n}(\tau)\right|$ achieves its maximum value. Let $j$ be such that $j T / n<\tau_{0} \leqq((j+1) T) / n$. By hypothesis

$$
\left|z\left(\frac{(j+1) T}{n}\right)-z\left(\frac{j T}{n}\right)\right| \leqq \frac{\delta}{2},
$$

and hence it follows that every point $\left(\tau, z^{n}(\tau)\right)$ on the straight line segment joining $(j T / n, z(j T / n))$ and $((j+1) T / n, z((j+1) T / n))$ satisfies $\left|z(j T / n)-z^{n}(\tau)\right| \leqq \delta / 2$. Again by hypothesis $\left|z(j T / n)-z^{n}(\tau)\right| \leqq \delta / 2$ so that by triangle inequality $\left|z^{n}(\tau)-z(\tau)\right|=\zeta$. This holds in particular for $\tau_{0}$ and the lemma is proved.

Lemma 3. $P\left\{\left\|z-z^{n}(\cdot)\right\| \geqq \gamma\right\} \leqq 4 / \gamma(2 n T / \pi)^{1 / 2} \exp \left(-n \gamma^{2} / 8 T\right)$.

Proof. Since Brownian motion has stationary increments we have from Lemma 1 that for any $j=0,1,2, \ldots, n-1$,

$$
\begin{aligned}
& P\left\{\sup _{j T / n<\tau \leqq(j+1) T / n}|z(\tau)-z(j T / n)| \geqq \gamma / 2\right\} \\
& \quad=P\left\{\sup _{0 \leqq \tau \leqq T / n}|z(\tau)| \geqq \gamma / 2\right\} \leqq 2(2 n / \pi T)^{1 / 2} \int_{\gamma / 2}^{\infty} \exp \left(-n u^{2} / 2 T\right) d u \\
&=2(2 / \pi)^{1 / 2} \int_{\gamma n^{1 / 2} / 2 T^{1 / 2}}^{\infty}\left(\exp \left(-v^{2} / 2\right)\right) d v \leqq 4 / \gamma(2 T / n \pi)^{1 / 2} \exp \left(-n \gamma^{2} / 8 T\right) .
\end{aligned}
$$

Let

$$
Q_{j}^{n}=\left\{\left.z \in C\right|_{j T / n<\tau \leqq(j+1) T / n} \sup _{\mid}|z(\tau)-z(j T / n)| \geqq \gamma / 2\right\}, \quad j=0,1,2, \ldots, n-1 .
$$

From the preceding inequality

$$
P\left\{\bigcup_{j=0}^{n-1} Q_{j}^{n}\right\} \leqq(4 / \gamma)(2 n T / \pi)^{1 / 2} \exp \left(-n \gamma^{2} / 8 T\right) .
$$

Now if $z \notin \bigcup_{j=0}^{n-1} Q_{j}^{n}$, then $\sup _{j T / n<\tau \leqq(j+1) T / n}|z(\tau)-z(j T / n)|<\gamma / 2$ for all $j=0,1, \ldots, n$ and hence from Lemma $2,\left\|z-z^{n}(\cdot)\right\| \leqq \gamma$. Thus

$$
P\left\{\left\|z-z^{n}(\cdot)\right\| \geqq \gamma\right\} \leqq(4 / \gamma)(2 n T / \pi)^{1 / 2} \exp \left(-n \gamma^{2} / 8 T\right) .
$$

LEMMA 4.

$$
\int_{0}^{T}\left[d s^{n}(\tau) / d \tau\right] d \tau=s^{n} A_{n}\left(s^{n}\right)^{-}
$$

and

$$
x^{n} A_{n}\left(x^{n}\right)^{-} \leqq \int_{0}^{T}\left[x^{\prime}(\tau)\right]^{2} d \tau \quad \text { for all } n,
$$


where $\left(x^{n}\right)^{-}$means $x^{n}$ transpose and

$$
A_{n}=\min _{1 \leqq i, j \leqq n}\left[\frac{i T}{n}, \frac{j T}{n}\right]^{-1} .
$$

Proof. By definition

$$
\int_{0}^{T}\left[\frac{d s^{n}(\tau)}{d \tau}\right]^{2} d \tau=\sum_{j=1}^{n} \frac{T}{n}\left[\frac{n}{T}\left(s_{j}^{n}-s_{j-1}^{n}\right)\right]^{2}
$$

where $s_{0}^{n}=0$. But as is well known

$$
\sum_{j=1}^{n} \frac{n}{T}\left[s_{j}^{n}-s_{j-1}^{n}\right]^{2}=s^{n} A_{n}\left(s^{n}\right)^{-}
$$

Now for the second part of the lemma. From the first part of this lemma, it is sufficient to show that

$$
\int_{0}^{T}\left[d x^{n}(\tau) / d \tau\right]^{2} d \tau \leqq \int_{0}^{T}\left[x^{\prime}(\tau)\right]^{2} d \tau
$$

Since

$$
\frac{d x^{n}(\tau)}{d \tau}=\frac{n}{T}\left(x\left(\frac{j T}{n}\right)-x\left(\frac{(j-1) T}{n}\right)\right) \quad \frac{j-1}{n}<\tau<\frac{j}{n}
$$

it follows from the Schwarz inequality, that

$$
\begin{aligned}
\int_{0}^{T}\left[\frac{d x^{n}(\tau)}{d \tau}\right]^{2} d \tau & =\sum_{j=1}^{n} \frac{n}{T}\left[\int_{(j-1) T / n}^{j T / n} x^{\prime}(\tau) d \tau\right]^{2} \\
& \leqq \sum_{j=1}^{n} \int_{(j-1) T / n}^{j T / n}\left[x^{\prime}(\tau)\right]^{2} d \tau \\
& =\int_{0}^{T}\left[x^{\prime}(\tau)\right]^{2} d \tau .
\end{aligned}
$$

LEMMA 5. If $z \in C^{*}[0, T]$, then for $\tau_{2}>\tau_{1}$

$$
\sup _{\tau_{1} \leqq \tau \leqq \tau_{2}}\left[z(\tau)-z\left(\tau_{1}\right)\right]^{2} \leqq\left(\tau_{2}-\tau_{1}\right) \int_{\tau_{1}}^{\tau_{2}}\left[z^{\prime}(\tau)\right]^{2} d \tau
$$

Proof. From the hypothesis of the Lemma it follows $z(\tau)-z\left(\tau_{1}\right)=\int_{\tau_{1}}^{\tau} z^{\prime}(s) d s$. From the Schwarz inequality it follows $\left[z(\tau)-z\left(\tau_{1}\right)\right]^{2} \leqq\left(\tau-\tau_{1}\right) \int_{\tau_{1}}^{\tau}\left[z^{\prime}(s)\right]^{2} d s$. The Lemma follows since the sup of $\left(\tau-\tau_{1}\right) \int_{\tau_{1}}^{\tau}\left[z^{\prime}(\tau)\right]^{2} d \tau$ for $\tau_{2} \geqq \tau \geqq \tau_{1}$, is taken on when $\tau=\tau_{2}$. 
Lemma 6. Suppose $F(z)$ satisfies the conditions of Theorem A. Then $A(\delta)<0$ if $\delta>0$, where $A(\delta)$ is defined by equation (2.1).

Proof. Suppose not. Then there is a sequence $\left\{z_{m}\right\} \subset C^{*}[0, T]$ such that

$$
\left\|z_{m}-x\right\| \geqq \delta \quad \text { and } \quad \lim _{m \rightarrow \infty} F\left(z_{m}\right)-\frac{1}{2} \int_{0}^{T}\left[z_{m}^{\prime}(\tau)\right]^{2} d \tau=b .
$$

It will be shown in the case of assumptions (3.1) above that the sequence $\left\{z_{m}\right\}$ has a subsequence $\left\{z_{m}^{*}\right\}$ which converges uniformly to $x^{*} \in C^{*}[0, T]$, that $x^{*} \neq x$, and that $F\left(x^{*}\right)-\frac{1}{2} \int_{0}^{T}\left[x^{* \prime}(\tau)\right]^{2} d \tau \geqq b$. This is contrary to the hypothesis of the Lemma.

It can clearly be assumed that $b-1 \leqq F\left(z_{m}\right)-\frac{1}{2} \int_{0}^{T}\left[z_{m}^{\prime}(\tau)\right]^{2} d \tau$ for all $m$. From Lemma 5 and condition 3 of Theorem A, it follows that $\int_{0}^{T}\left[z_{m}^{\prime}(\tau)\right]^{2} d \tau \leqq 4\left|L_{1}-b+1\right|$. From Lemma 5 again, it follows that

$$
\begin{aligned}
\sup _{\tau_{1} \leqq \tau \leqq \tau_{2}}\left|z_{m}(\tau)-z_{m}\left(\tau_{1}\right)\right| & \leqq\left[\left(\tau_{2}-\tau_{1}\right) \int_{\tau_{1}}^{\tau_{2}}\left[z_{m}^{\prime}(\tau)\right]^{2} d \tau\right]^{1 / 2} \\
& \leqq 2\left[\left(\tau_{2}-\tau_{1}\right)\left|L_{1}-b+1\right|\right]^{1 / 2}
\end{aligned}
$$

Therefore, the sequence $\left\{z_{m}\right\}$ is equicontinuous and bounded. By Ascoli's theorem it follows that there exists $x^{*} \in C^{*}[0, T]$ and a subsequence $\left\{z_{m}^{*}\right\}$ such that $x^{*}$ is the uniform limit of $\left\{z_{m}^{*}\right\}$. From the inequality $b-1 \leqq F\left(z_{m}\right)-\frac{1}{2} \int_{0}^{T}\left[z_{m}^{\prime}(\tau)\right]^{2} d \tau$ and conditions 3 and 5 of Theorem $\mathrm{A}$ it also follows that $F(z)$ is continuous at $x^{*}$. Since $\lim \inf \int_{0}^{T}\left[z_{m}^{* \prime}(\tau)\right]^{2} d \tau \geqq \int_{0}^{T}\left|x^{*^{\prime}}(\tau)\right|^{2} d \tau$ is a standard property of weak convergence, it follows that

$$
F\left(x^{*}\right)-\frac{1}{2} \int_{0}^{T}\left[x^{* \prime}(\tau)\right]^{2} d \tau \geqq \lim \inf F\left(z_{m}\right)-\frac{1}{2} \int_{0}^{T}\left[z_{m}^{\prime}(\tau)\right]^{2} d \tau \geqq b
$$

LEMMA 7. If $\left\|s^{n}-x^{n}\right\| \geqq w$ and $w-p_{n}>0$, then $F\left(s^{n}\right)-\frac{1}{2} s^{n} A_{n}\left(s^{n}\right)^{-} \leqq A\left(w-p_{n}\right)+b$, where $p_{n}=\left\|x-x^{n}(\cdot)\right\| ; x^{n}(\cdot)$ is the polygonalization of the maximizing function $x$.

Proof. $\left\|x^{n}(\cdot)-s^{n}(\cdot)\right\| \leqq\left\|x-s^{n}(\cdot)\right\|+\left\|x-x^{n}(\cdot)\right\|$. Thus

$$
\begin{aligned}
\left\|x-s^{n}(\cdot)\right\| & \geqq\left\|x^{n}(\cdot)-s^{n}(\cdot)\right\|-\left\|x-x^{n}(\cdot)\right\| \\
& =\left\|x^{n}(\cdot)-s^{n}(\cdot)\right\|-p_{n}=\left\|x^{n}-s^{n}\right\|-p_{n} \geqq w-p_{n} .
\end{aligned}
$$

By Lemma $4, s^{n} A_{n}\left(s^{n}\right)^{-}=\int_{0}^{T}\left[d s^{n}(\tau) / d \tau\right]^{2} d \tau$. Thus

$$
F\left(s^{n}\right)-\frac{1}{2} s^{n} A_{n}\left(s^{n}\right)^{-}=F\left(s^{n}(\cdot)\right)-\frac{1}{2} \int_{0}^{T}\left[\frac{d s^{n}(\tau)}{d \tau}\right]^{2} d \tau .
$$


By the definition of $A(\delta), b$ and Lemma 6 , and since $\left\|x-s^{n}(\cdot)\right\| \geqq w-p_{n}$ we have

$$
F\left(s^{n}\right)-\frac{1}{2} s^{n} A_{n}\left(s^{n}\right)^{-} \leqq A\left(w-p_{n}\right)+b .
$$

LEMmA 8. If $F(z)$ satisfies the hypothesis of Theorem $\mathrm{A}$, and $B$ is any number greater than zero, then for all $\lambda$ sufficiently small

$$
I_{1}(\lambda)=\exp \left(-b \lambda^{-2}\right) E_{z}^{w}\left\{\exp \left(\lambda^{-2} F(\lambda z)\right)\right\} \geqq \frac{1}{4} \exp \left(-B \lambda^{-2}\right) .
$$

Proof. The translation formula for Wiener integrals [1] states that if $q_{0}(\tau) \in$ $C^{*}[0, T]$ and $H(q)$ is a Wiener measurable functional, then

$$
E_{q}^{w}\left[H\left(q_{0}+q_{0}\right)\right]=\exp \left(-\frac{1}{2} \int_{0}^{T}\left[q_{0}^{\prime}(\tau)\right]^{2} d \tau\right) E_{y}^{w}\left[H(y) \exp \left[\int_{0}^{T} q_{0}^{\prime}(\tau) d y(\tau)\right]\right] .\left(^{5}\right)
$$

Because of the assumption made on $x(\tau)$ it follows, using this translation formula, that

$$
\begin{aligned}
& I_{1}=\exp {\left[\left(-\frac{1}{2} \lambda^{-2}\right) \int_{0}^{T}\left[x^{\prime}(\tau)\right]^{2} d \tau\right] } \\
& \times E_{y}^{w}\left\{\exp \left[\lambda^{-2}[F(\lambda y+x)-b]-\lambda^{-1} \int_{0}^{T} x^{\prime}(\tau) d y(\tau)\right]\right\}
\end{aligned}
$$

From the continuity assumption of $F(z)$, it follows that there exists a $\beta>0$ such that $\|x-y\| \leqq \beta$ implies $F(x) \leqq F(y)+B$. Thus, from (3.3)

$$
\begin{aligned}
I_{1} \geqq & \exp \left[\left(-\frac{1}{2} \lambda^{-2}\right) \int_{0}^{T}\left[x^{\prime}(\tau)\right]^{2} d \tau\right] \\
& \times E_{y}^{w}\left\{\chi(\beta / \lambda, 0, y) \exp \left[\lambda^{-2}[F(\lambda y+x)-b]-\lambda^{-1} \int_{0}^{T} x^{\prime}(\tau) d y(\tau)\right]\right\} \\
\geqq & \exp \left[\left(-\frac{1}{2} \lambda^{-2}\right) \int_{0}^{T}\left[x^{\prime}(\tau)\right]^{2} d \tau\right] \\
& \times E_{y}^{w}\left\{\chi(\beta / \lambda, 0, y) \exp \left[\lambda^{-2}(F(x)-B-b)-\lambda^{-1} \int_{0}^{T} x^{\prime}(\tau) d y(\tau)\right]\right\},
\end{aligned}
$$

where $\chi(\delta, y, z)$ is the characteristic function of the set $\{z \in C \mid\|y-z\| \leqq \delta\}$. But by assumption on $x(\tau), F(x)=\frac{1}{2} \int_{0}^{T}\left[x^{\prime}(\tau)\right]^{2} d \tau+b$ and, therefore,

$$
I_{1} \geqq \exp \left(-B \lambda^{-2}\right) E_{y}^{w}\left\{\chi(\beta / \lambda, 0, y) \exp \left(\lambda^{-1} \int_{0}^{T} x^{\prime}(\tau) d y(\tau)\right)\right\}
$$

$\left(^{5}\right)$ See [4, Chapter IX], for a definition of stochastic integral. 
Now let $R(y)$ be the characteristic function of the set

$$
\left\{y \in C \mid \int_{0}^{T} x^{\prime}(\tau) d y(\tau) \leqq 0\right\} .
$$

From (3.4)

$$
I_{1} \geqq \exp \left(-B \lambda^{-2}\right) E_{y}^{w}\{\chi(\beta / \lambda, 0, y) R(y)\} .
$$

But by the symmetry of the Wiener measure with respect to reflections across the $\tau$ axis,

$$
E_{y}^{w}\{\chi(\beta / \lambda, 0, y) R(y)\} \geqq \frac{1}{2} E_{y}^{w}\{\chi(\beta / \lambda, 0, y)\}
$$

Therefore

$$
I_{1} \geqq \frac{1}{2} \exp \left(-B \lambda^{-2}\right) E_{y}^{w}\{\chi(\beta / \lambda, 0, y)\}=\frac{1}{2} \exp \left(-B \lambda^{-2}\right) P\{\|y\| \leqq \beta / \lambda\} .
$$

Since $P\{\|y\| \leqq \beta / \lambda\} \rightarrow 1$ as $\lambda \rightarrow 0$, it finally follows for small enough $\lambda$ that

$$
I_{1} \geqq \frac{1}{4} \exp \left(-B \lambda^{-2}\right) \text {. }
$$

LEMMA 9. If $F(z)$ and $G(z)$ satisfy the hypothesis of Theorem $\mathrm{A}$ and $\delta$ is any positive number, then

$$
\begin{aligned}
I(\lambda) & =\exp \left(-b \lambda^{-2}\right) E_{z}^{w}\left\{[1-\chi(\delta / \lambda, x / \lambda, z)]|G(\lambda z)| \exp \left(\lambda^{-2} F(\lambda z)\right)\right\} \\
& =0\left(\exp \left(\lambda^{-2} \alpha\right)\right)
\end{aligned}
$$

where $\alpha<0$.

Proof. Since $x(\tau)$ is continuous on $0 \leqq \tau \leqq T$, we have

$$
\lim _{n \rightarrow \infty}\left\|x-x^{n}(\cdot)\right\|=0
$$

and from Lemma 4 we have

$$
\lim _{n \rightarrow \infty} x^{n} A_{n}\left(x^{n}\right)^{-} \leqq \int_{0}^{T}\left[x^{\prime}(\tau)\right]^{2} d \tau .
$$

Therefore, both sequences $\left\|x^{n}\right\|$ and $x^{n} A_{n}\left(x^{n}\right)^{-}$are bounded. Hence there exists a positive constant $c$ so large that for all $n$

$$
L_{1} / c+2 L_{2}\left\|x^{n}\right\|(T / c)^{1 / 2}+L_{2}\left(\left\|x^{n}\right\|^{2}\right) / c+\left(x^{n} A_{n}\left(x^{n}\right)^{-} / c\right)^{1 / 2} \leqq \frac{1}{8} .
$$

In addition, $c$ can be chosen so that

$$
(-b-A(\delta)) / c \leqq \frac{1}{8}
$$


From the continuity assumption on $F(z)$, it follows that there exists $0<\eta<\delta / 4$ such that

$$
\|z\|<\left[\left|b-L_{1}+1\right| /\left|2 L_{2}-1 / 2 T\right|\right]^{1 / 2} \text { and }\|z-y\|=\eta
$$

imply

$$
F(z)-F(y) \leqq-A(\delta / 2)(\delta / 2)^{2} / 4 T c .
$$

Finally, let $n$ be a positive integer so large that

$$
L_{1}-b-n \eta^{2} / 16 T \leqq-1
$$

and $\left\|x-x^{n}(\cdot)\right\| \leqq \delta / 8$, i.e., $p_{n} \leqq \delta / 8$. In what follows keep these choices of $c, \eta$, and $n$ fixed.

For convenience, the following notation is introduced. Let

$$
a=\left[\left|b-L_{1}+1\right| /\left|2 L_{2}-1 / 2 T\right|\right]^{1 / 2}
$$

and let

$$
\begin{aligned}
I_{2} & =I_{2}(\delta, \eta, n, \lambda) \\
& =E_{z}^{w}\left\{[1-\chi(\delta / \lambda, x / \lambda, z)][1-H(\eta / \lambda, n, z)]|G(\lambda z)|\left(\exp \lambda^{-2}[F(\lambda z)-b]\right)\right\}
\end{aligned}
$$

where $H(\eta, n, z)$ is the characteristic function of the set

$$
\left\{z \in C^{*}[0, T] \mid\left\|z-z^{n}\right\| \leqq \eta\right\}
$$

$$
\begin{aligned}
I_{3} & =I_{3}(\delta, \eta, n, \lambda) \\
& =E_{z}^{w}\left\{[1-\chi(\delta / \lambda, x / \lambda, z)] H(\eta / \lambda, n, z) \chi(a / \lambda, 0, z)|G(\lambda z)|\left(\exp \lambda^{-2}[F(\lambda z)-b]\right)\right\} . \\
I_{4} & =I_{4}(\delta, \eta, n, \lambda) \\
& =E_{z}^{w}\left\{[[1-\chi(\delta / \lambda, x / \lambda, z)] H(\eta / \lambda, n, z)][1-\chi(a / \lambda, 0, z)]|G(\lambda z)|\left(\exp \lambda^{-2}[F(\lambda z)-b]\right)\right\} .
\end{aligned}
$$

It will be shown that $I_{2}, I_{3}$, and $I_{4}$ are $0\left(\exp \left(\alpha \lambda^{-2}\right)\right)$ where $\alpha<0$. Since $I=$ $I_{2}+I_{3}+I_{4}$ this will imply the conclusion of the lemma. First consider $I_{2}$. By hypothesis, for almost all $z$,

$$
|G(z)| \leqq K_{1} \exp \left(K_{2}\|z\|^{2}\right) \quad \text { where } \quad K_{1}>0, \quad K_{2}>0 .
$$

Thus

$$
I_{2} \leqq K_{1} E_{z}^{w}\left\{[1-H(\eta / \lambda, n, z)] \exp \left(\lambda^{2} K_{2}\|z\|^{2}+\lambda^{-2}[F(\lambda z)-b]\right)\right\}
$$

Applying the Schwarz inequality,

$$
\begin{aligned}
\left|I_{2}\right| \leqq & K_{1}\left(E_{z}^{w}\{1-H(\eta / \lambda, n, z)\}\right)^{1 / 2} \\
& \times\left(E_{z}^{w}\left\{\exp \left(2 \lambda^{2} K_{2}\|z\|^{2}+\lambda^{-2}[F(\lambda z)-b]\right)\right\}\right)^{1 / 2}
\end{aligned}
$$


From Lemma 3,

$$
\begin{aligned}
E_{z}^{w}\{[1-H(\eta / \lambda, n, z)]\} & \left.=P\left\{\left\|z-z^{n}(\cdot)\right\| \geqq \eta / \lambda\right)\right\} \\
& \leqq(4 \lambda / \eta)(2 n T / \pi)^{1 / 2} \exp \left(-n \eta^{2} / 8 \lambda^{2} T\right) .
\end{aligned}
$$

By hypothesis, $F(\lambda z) \leqq L_{1}+\lambda^{2} L_{2}\|z\|^{2}$ where $L_{1}>0$ and $L_{2}<\frac{1}{4} T$. Hence, from Lemma 1,

$$
\begin{aligned}
& E_{z}^{w}\left\{\exp \left(2 \lambda^{2} K_{2}\|z\|^{2}+2 \lambda^{-2}[F(\lambda z)-b]\right)\right\} \\
& \quad \leqq \exp \left(\lambda^{-2}\left(2 L_{1}-2 b\right)\right) E_{z}^{w}\left\{\exp \left(\left(2 \lambda^{2} K_{2}+2 L_{2}\right)\|z\|^{2}\right)\right\} \\
& \quad \leqq 2 \exp \left[\left(2 L_{1}-2 b\right) \lambda^{-2}\right]\left(\left(\frac{2}{\pi T}\right)^{1 / 2} \int_{0}^{\infty} \exp \left[\left(2 \lambda^{2} K_{2}+2 L_{2}\right) u^{2}-u^{2} / 2 T\right] d u\right) .
\end{aligned}
$$

Since $L_{2}<\frac{1}{4} T$, it follows that for sufficiently small $\lambda$ the integral on the right of (3.11) converges, and therefore for sufficiently small $\lambda$

$$
I_{2}=P \exp \left(-n \eta^{2} / 16 \lambda^{2} T+\left(L_{1}-b\right) \lambda^{-2}\right)
$$

where $P$ is a constant. From choice of $n$ in (3.8)

$$
I_{2} \leqq P \exp \left(-\lambda^{-2}\right) \text {. }
$$

Consider now $I_{3}$.

$$
\begin{array}{r}
I_{3} \leqq K_{1} E_{z}^{w}\left\{\left[1-\chi\left(\frac{\delta}{\lambda}, \frac{x}{\lambda}, z\right)\right] H\left(\frac{\eta}{\lambda}, n, z\right) \chi\left(\frac{a}{\lambda}, 0, z\right)\right. \\
\left.\cdot \exp \left(\lambda^{2} K_{2}\|z\|^{2}+\lambda^{-2}[F(\lambda z)-b]\right)\right\} .
\end{array}
$$

Set $D=-A(\delta / 2)(\delta / 2)^{2} / 4 T c$ (cf. (3.7)).

In the integral on the right of (3.13) the integration is over the set

$$
\left\{z \in C \mid\left\|\lambda z-\lambda z^{n}(\cdot)\right\| \leqq \eta \text { and }\|z\| \leqq a / \lambda\right\}
$$

and hence by the choice of $\eta$ it follows that

$$
F(\lambda z) \leqq F\left(\lambda z^{n}(\cdot)\right)+D
$$

Also $\left\|z-z^{n}(\cdot)\right\| \leqq \eta / \lambda$ implies

$$
\|z\|^{2} \leqq\left\|\left|z^{n}(\cdot)\right|+\frac{\eta}{\lambda}\right\|^{2}
$$

Using (3.14) and (3.15), in (3.13), one obtains

$$
\begin{aligned}
I_{3} \leqq K_{1} E_{z}^{w}\{ & {[1-\chi(\delta / \lambda, x / \lambda, z)] H(\eta / \lambda, n, z) } \\
\cdot & \left.\exp \left[\lambda^{2} K_{2}\left\|\left|z^{n}(\cdot)\right|+\eta / \lambda\right\|^{2}+\lambda^{-2}\left(F\left(\lambda z^{n}(\cdot)\right)+D-b\right)\right]\right\} .
\end{aligned}
$$


Now

$\left\{z \in C \mid\left\|\lambda z-\lambda z^{n}(\cdot)\right\| \leqq \eta,\|\lambda z-x\|>\delta\right\} \subset\left\{z \in C \mid\left\|\lambda z^{n}(\cdot)-x\right\|>\delta-\eta\right\}$,

and therefore

$$
\begin{aligned}
I_{3} \leqq K_{1} E_{z}^{w}\left\{\left[1-\chi((\delta-\eta) / \lambda), x / \lambda, z^{n}(\cdot)\right)\right] \\
\left.\cdot \exp \left[\lambda^{2} K_{2}\left\|\left|z^{n}(\cdot)\right|+\eta / \lambda\right\|^{2}+\lambda^{-2}\left(F\left(\lambda z^{n}(\cdot)\right)+D-b\right)\right]\right\}
\end{aligned}
$$

The integrand on the right of (3.17) is now a functional depending only on a finite number of $\tau$ points; namely, it is a function of $z(T / n), z(2 T / n), \ldots, z(T)$, and hence the function space integral reduces to an $n$-dimensional integral. Thus

$$
\begin{aligned}
I_{3} \leqq & \frac{K_{1} \exp \left(-b \lambda^{-2}\right)}{\left((2 \pi)^{n}(T / n)^{n}\right)^{1 / 2}} \int_{R_{n}}\left[1-\chi\left((\delta-\eta) / \lambda, x / \lambda, z^{n}(\cdot)\right)\right] \\
& \cdot \exp \left(\lambda^{-2} K_{2}\left\|\left|z^{n}\right|+\frac{\eta}{\lambda}\right\|^{2}+\lambda^{-2}\left(F\left[\lambda z^{n}\right]+D\right)-\frac{1}{2} z^{n} A_{n}\left(z^{n}\right)^{-}\right) d z^{n}
\end{aligned}
$$

Letting $z^{n}=\lambda^{-1}\left(w^{n}+x^{n}\right)$ one sees that

$$
\begin{aligned}
I_{3} \leqq & {\left[\lambda^{-n} K_{1} \exp \left((D-b) \lambda^{-2}\right)\right](2 \pi T / n)^{-n / 2} \int_{R_{n}}\left[1-\chi\left(\delta-\eta, x, w^{n}+x^{n}(\cdot)\right)\right] } \\
& \cdot \exp \left(K_{2}\left\|\left|w^{n}+x^{n}\right|+\eta\right\|^{2}+\lambda^{-2} F\left[w^{n}+x^{n}\right]-\frac{1}{2} \lambda^{-2}\left[w^{n}+x^{n}\right] A_{n}\left[w^{n}+x^{n}\right]^{-}\right) d w^{n} .
\end{aligned}
$$

Since $n$ was picked so that $p_{n}=\left\|x^{n}-x\right\| \leqq \delta / 8$ and $\eta<\delta / 4$, it follows that $\delta-\eta-p_{n}>$ $\delta-\eta-2 p_{n}>0$.

Clearly $\left\|w^{n}\right\| \leqq \delta-\eta-p_{n}$ implies $\left\|w^{n}(\cdot)+x^{n}(\cdot)-x\right\| \leqq \delta-\eta$ and hence

$$
\begin{aligned}
1-\chi\left(\delta-\eta, x, w^{n}+x^{n}(\cdot)\right) & \leqq 1-\chi\left(\delta-\eta-p_{n}, 0, w^{n}(\cdot)\right) \\
= & 1-\text { characteristic function of }\left\{w^{n} \in R^{n} \mid\left\|w^{n}\right\| \leqq \delta-\eta-p_{n}\right\}
\end{aligned}
$$

Therefore

$$
\begin{aligned}
I_{3} \leqq\left[K_{1} \lambda^{-n} \exp (D-b) \lambda^{-2}\right] \cdot(2 \pi T / n)^{-n / 2} \\
\quad \int_{\left\|w^{n}\right\| \geqq \delta-n-p_{n}} \exp \left(K_{2}\left\|\left|w^{n}+x^{n}\right|+\eta\right\|^{2}+w^{n} A_{n}\left(w^{n}\right)^{-\lambda^{-2}}\right. \\
\left.\quad \times\left[\frac{F\left[w^{n}+x^{n}\right]-\frac{1}{2}\left[w^{n}+x^{n}\right] A_{n}\left[w^{n}+x^{n}\right]^{-}}{w^{n} A_{n}\left(w^{n}\right)^{-}}\right]\right) d w^{n} .
\end{aligned}
$$

In the integral on the right of (3.18) the integration is over those points in $R_{n}$ where $\left\|w^{n}\right\| \geqq \delta-\eta-p_{n}$. It will be shown now that over that set

$$
J \equiv \frac{F\left[w^{n}+x^{n}\right]-\frac{1}{2}\left[w^{n}+x^{n}\right] A_{n}\left[w^{n}+x^{n}\right]^{-}}{w^{n} A_{n}\left(w^{n}\right)^{-}} \leqq \frac{A\left(\delta-\eta-2 p_{n}\right)+b}{c}
$$


where $A(\delta)$ is defined in (2.1) and $c$ is the constant picked in (3.5) and (3.6). This can be seen by considering the two possibilities $w^{n} A_{n}\left(w^{n}\right)^{-}<c$ and $w^{n} A_{n}\left(w^{n}\right)^{-} \geqq c$. In the first case, it follows from Lemma 7 that (remembering $\left(\delta-\eta-2 p_{n}>0\right)$ ),

$$
F\left(w^{n}+x^{n}\right)-b-\frac{1}{2}\left(w^{n}+x^{n}\right) A_{n}\left(w^{n}+x^{n}\right)^{-} \leqq A\left(\delta-\eta-2 p_{n}\right),
$$

and therefore

$$
J \leqq \frac{A\left(\delta-\eta-2 p_{n}\right)+b}{c} .
$$

In the second case $\left(w^{n} A_{n}\left(w^{n}\right)^{-} \geqq c\right)$ it follows from the hypothesis on $F(z), L_{2}<\frac{1}{4} T$, and Lemmas 4 and 5 that

$$
\begin{aligned}
J & \leqq \frac{L_{1}+L_{2}\left\|w^{n}+x^{n}\right\|^{2}-\frac{1}{2}\left(w^{n}+x^{n}\right) A_{n}\left(w^{n}+x^{n}\right)^{-}}{w^{n} A_{n}\left(w^{n}\right)^{-}} \\
& \leqq \frac{L_{1}+L_{2}\left\|w^{n}\right\|^{2}+2 L_{2}\left\|w^{n}\right\|\left\|x^{n}\right\|+L_{2}\left\|x^{n}\right\|^{2}-\frac{1}{2} w^{n} A_{n}\left(w^{n}\right)^{-}-w^{n} A_{n}\left(x^{n}\right)^{-}-\frac{1}{2} x^{n} A_{n}\left(x^{n}\right)^{-}}{w^{n} A_{n}\left(w^{n}\right)^{-}} .
\end{aligned}
$$

Since $-\frac{1}{2} x^{n} A_{n}\left(x^{n}\right)^{-} \leqq 0$,

$$
\begin{aligned}
J & \left.\leqq \frac{L_{1}}{w^{n} A_{n}\left(w^{n}\right)^{-}}+L_{2} T+2 L_{2}\left\|x^{n}\right\|\left(\frac{T}{w^{n} A_{n}\left(w^{n}\right)^{-}}\right)^{1 / 2}+L_{2} \frac{\left\|x^{n}\right\|^{2}}{w^{n} A_{n}\left(w^{n}\right)^{-}}-\frac{1}{2}-\frac{w^{n} A_{n}\left(x^{n}\right)^{-}}{w^{n} A_{n}\left(w^{n}\right)^{-}}\right) \\
& \leqq-\frac{1}{4}+L_{1} / c+2 L_{2}\left\|x^{n}\right\|(T / c)^{1 / 2}+L_{2}\left\|x^{n}\right\|^{2} / c+\left(\frac{x^{n} A_{n}\left(x^{n}\right)^{-}}{c}\right)^{1 / 2}
\end{aligned}
$$

where in the last inequality, the bound

$$
w^{n} A_{n}\left(x^{n}\right)^{-} \leqq\left[\left(w^{n} A_{n}\left(w^{n}\right)^{-}\right)\left(x^{n} A_{n}\left(x^{n}\right)^{-}\right)\right]^{1 / 2}
$$

was used. But from (3.5) and (3.6) it follows that

$$
J \leqq-1 / 8 \leqq(A(\delta)+b) / c \leqq\left(A\left(\delta-\eta-2 p_{n}\right)+b\right) / c,
$$

which completes the proof of (3.19). Now using (3.19) in (3.18) it can be seen that

$$
\begin{aligned}
I_{3} \leqq & {\left[K_{1} \lambda^{-n} \exp \left(D \lambda^{-2}\right)\right](2 \pi T / n)^{-n / 2} } \\
& \cdot \int_{\left\|w^{n}\right\| \geqq\left(\delta-\eta-p_{n}\right)} \exp \left(K_{2}\left\|\left|w^{n}+x^{n}\right|+\eta\right\|^{2}\right. \\
& \left.+\frac{1}{2} \lambda^{-2} w^{n} A_{n}\left(w^{n}\right)^{-}\left[2 A\left(\delta-\eta-2 p_{n}\right) / c\right]\right) d w^{n} .
\end{aligned}
$$

Let $v=\left(-2 A\left(\delta-\eta-2 p_{n}\right) / c\right)^{1 / 2}$ and $\mu^{n}=(v / \lambda) w^{n}$, giving

$$
\begin{aligned}
I_{3} \leqq & {\left[K_{1} v^{-n} \exp \left(D \lambda^{-2}\right)\right](2 \pi T / n)^{-n / 2} } \\
& \cdot \int_{\left\|u^{n}\right\|=\left(\delta-\eta-p_{n}\right) v / \lambda} \exp \left(K_{2}\left\|\left|\lambda \mu^{n} / v+x^{n}\right|+\eta\right\|^{2}-\frac{1}{2} \mu^{n} A_{n}\left(\mu^{n}\right)^{-}\right) d \mu^{n} .
\end{aligned}
$$

$\left.{ }^{6}\right)$ It can be seen that $w^{n} A_{n}\left(w^{n}\right)^{-} \geqq(1 / T)\left\|w^{n}\right\|^{2}$. 
Changing back to the function space integral, this gives

$$
\begin{aligned}
I_{3} \leqq K_{1} v^{-n} \exp \left(D \lambda^{-2}\right) E_{z}^{w}\left\{\left[1-\chi\left(\left(\delta-\eta-p_{n}\right) v / \lambda, 0, z^{n}(\cdot)\right)\right]\right. \\
\left.\cdot \exp \left(K_{2}\left\|\left|\lambda z^{n} / v+x^{n}\right|+\eta\right\|^{2}\right)\right\} .
\end{aligned}
$$

But clearly

$$
1-\chi\left(\left(\delta-\eta-p_{n}\right) v / \lambda, 0, z^{n}(\cdot)\right) \leqq 1-\chi\left(\left(\delta-\eta-p_{n}\right) v / \lambda, 0, z\right)
$$

and thus from (3.20) and the Schwarz inequality

$$
\begin{aligned}
I_{3} \leqq & K_{1} v^{-n} \exp \left(D \lambda^{-2}\right) E_{z}^{w}\left\{\left[1-\chi\left(\left(\delta-\eta-p_{n}\right) v / \lambda\right), 0, z\right]\right\}^{1 / 2} \\
& \times E_{z}^{w}\left\{\exp \left[2 K_{2}\left\|\left|\lambda z^{n} / v+x^{n}\right|+\eta\right\|^{2}\right]\right\}^{1 / 2}
\end{aligned}
$$

From Lemma 1,

$$
\begin{aligned}
E_{z}^{w}\left\{\left[1-\chi\left(\left(\delta-\eta-p_{n}\right) v / \lambda, 0, z\right)\right]\right\} & \leqq 2\left(\frac{2}{\pi T}\right)^{1 / 2} \int_{\left(\delta-\eta-p_{n}\right) v / \lambda}^{\infty} \exp \left(-u^{2} / 2 T\right) d u \\
& \leqq \text { const. } \cdot \exp \left(-\left(\delta-\eta-p_{n}\right)^{2} v^{2} / 2 \lambda^{2} T\right)
\end{aligned}
$$

Since $\left\|z^{n}\right\| \leqq\|z\|$, we have from the triangle inequality

$$
E_{z}^{w}\left\{\exp \left(2 K_{2}\left\|\left|\lambda z^{n} / v+x^{n}\right|+\eta\right\|^{2}\right)\right\} \leqq \text { const. } E_{z}^{w}\left\{\exp \left(\lambda^{2} \text { const. }\|z\|^{2}\right)\right\}<\infty
$$

for small $\lambda$. Thus for small enough $\lambda$

$$
I_{3} \leqq \exp \left(\left(\delta-\eta-p_{n}\right)^{2} v^{2} /-4 \lambda^{2} T+D / \lambda^{2}\right) .
$$

Since

$$
0<D=-A(\delta / 2)(\delta / 2)^{2} / 4 T c,
$$

we have for sufficiently small $\lambda$, from (3.23) and the definition of $v$ and $D$,

$$
I_{3} \leqq \text { const. } \exp \left(\frac{\left(\delta-\eta-p_{n}\right)^{2} A\left(\delta-\eta-2 p_{n}\right)}{2 \lambda^{2} T c}-\frac{A(\delta / 2)(\delta / 2)^{2}}{4 \lambda^{2} T c}\right)
$$

Since $\eta=\delta / 4, p_{n}=\delta / 8$ and $A(\delta)$ is a decreasing function,

$$
I_{3} \leqq \text { const. } \exp \left(\frac{A(\delta / 2)(\delta / 2)^{2}}{2 \lambda^{2} T c}-\frac{A(\delta / 2)(\delta / 2)^{2}}{4 \lambda^{2} T c}\right)
$$

or

$$
I_{3} \leqq \text { const. } \exp \left(\frac{A(\delta / 2)(\delta / 2)^{2}}{4 \lambda^{2} T c}\right)
$$


Consider now $I_{4}$. Clearly,

$$
\left.I_{4} \leqq K_{1} E_{z}^{w}\left\{[1-\chi(a / \lambda, 0, z)] \exp \left[\left(L_{1}-b\right) \lambda^{-2}+K_{2} \lambda^{2}+L_{2}\right)\|z\|^{2}\right]\right\}
$$

By Lemma 1,

$$
I_{4} \leqq K_{1} \exp \left[\left(L_{1}-b\right) \lambda^{-2}\right] 2\left(\frac{2}{\pi T}\right)^{1 / 2} \int_{a / \lambda}^{\infty} \exp \left[\left(K_{2} \lambda^{2}+L_{2}-\frac{1}{2 T}\right) \mu^{2}\right] d u,
$$

for $K_{2} \lambda^{2} \leqq L_{2}$

$$
I_{4} \leqq K_{1} \exp \left[\left(L_{1}-b\right) \lambda^{-2}\right] 2\left(\frac{2}{\pi T}\right)^{1 / 2} \int_{a / \lambda}^{\infty} \exp \left[\left(2 L_{2}-1 / 2 T\right) \mu^{2}\right] d u .
$$

Remembering the definition of $a$, we find that $I_{4} \leqq K_{4} \exp \left(\lambda^{-2}\right)$ where $K_{4}$ is an absolute constant.

This proves Lemma 9, with

$$
\alpha=\min \left(-1, \frac{A(\delta / 2)(\delta / 2)^{2}}{4 T c}\right)
$$

LEMMA $10\left(^{7}\right)$. If $x^{\prime}(\tau)$ is of bounded variation and if

$$
\int_{0}^{T} f(\tau) y(\tau) d \tau-\int_{0}^{T} x^{\prime}(\tau) d y(\tau)=0
$$

for $f \in L^{2}[0, T]$, and for $y \in C^{*}[0, T]$, then

$$
\int_{0}^{T} f(\tau) y(\tau) d \tau-\int_{0}^{T} x^{\prime}(\tau) d y(\tau)=0
$$

for $y \in C[0, T]$.

Proof. Define $\int_{0}^{T} x^{\prime}(\tau) d y(\tau)$ by $-\int_{0}^{T} y(\tau) d x^{\prime}(\tau)+x^{\prime}(T) y(T)$. Then $\int_{0}^{T} f(\tau) y(\tau) d \tau$ $-\int_{0}^{T} x^{\prime}(\tau) d y(\tau)$ is obviously a continuous functional on $C$.

4. Proof of main theorems. In this section the proofs of Theorems A, B, and $\mathrm{C}$ will be given.

Proof of Theorem A. Let $\varepsilon>0$ be given. Choose $\delta$ so that $|G(y)-G(x)| \leqq \varepsilon / 2$ if $\|y-x\| \leqq \delta$. This is possible since $G(x)$ is continuous at $x$.

One sees that

(7) See [4, Chapter IX], for a definition of stochastic integrals. 


$$
\begin{aligned}
& \left|\frac{E_{z}^{w}\left\{G(\lambda z) \exp \left(\lambda^{-2} F(\lambda z)\right)\right\}}{E_{z}^{w}\left\{\exp \left(\lambda^{-2} F(\lambda z)\right)\right\}}-G(x)\right| \\
& \leqq \\
& \quad \frac{E_{z}^{w}\left\{\chi(\delta / \lambda, x / \lambda, z)|G(\lambda z)-G(x)| \exp \left(\lambda^{-2} F(\lambda z)\right)\right\}}{E_{z}^{w}\left\{\exp \left(\lambda^{-2} F(\lambda z)\right)\right\}} \\
& \quad+\frac{\exp \left(-b \lambda^{-2}\right) E_{z}^{w}\left\{[1-\chi(\delta / \lambda, x / \lambda, z)]|G(\lambda z)-G(x)| \exp \left(\lambda^{-2} F(\lambda z)\right)\right\}}{\exp \left(-b \lambda^{-2}\right) E_{z}^{w}\left\{\exp \left(\lambda^{-2} F(\lambda z)\right)\right\}}
\end{aligned}
$$

From Lemma 9 it follows that the numerator of the second term on the right of (4.1) is $0\left(\exp \left(\alpha \lambda^{-2}\right)\right)$ where $\alpha<0$. Choose B of Lemma 8 to be $\alpha / 2$ and apply Lemma 8 to the denominator of the second term of (4.1). Thus the second term on the right of $(4.1)$ is $0\left(\exp \left(\alpha \lambda^{-2} / 2\right)\right)$ and thus for sufficiently small $\lambda$ can be made less than $\varepsilon / 2$. The first term is less than $\varepsilon / 2$ by choice of $\delta$. Thus the left hand side of (4.1) is less than $\varepsilon$. This proves Theorem A.

Proof of Theorem B. Choose $\gamma>0$, then for all $\lambda$ sufficiently small we will show that

$$
\left|\exp \left(-b \lambda^{-2}\right) E_{z}^{w}\left\{G(\lambda z) \exp \left(\lambda^{-2} F(\lambda z)\right)\right\}-G(x) E_{z}^{w}\left\{\exp \left(f_{2}(0) z^{2}\right)\right\}\right| \leqq 4 \gamma .
$$

Pick a $\delta>0$ so that

i. The first two Frechet derivatives of $F(z)$ exist in a $\delta$ uniform neighborhood of $x$.

ii. $\sup _{\|\eta\|,\|y\| \leqq \delta}|G(y+x)-G(x)| E_{z}^{w}\left\{\exp \left(f_{2}(\eta) z^{2}\right)\right\} \leqq \gamma$.

iii. $|G(x)| E_{z}^{w}\left\{\left|\exp \left(f_{2}(\eta) z^{2}\right)-\exp \left(f_{2}(0) z^{2}\right)\right|\right\} \leqq \gamma$ if $\|\eta\| \leqq \delta$.

Let

$$
h_{1}(\lambda)=E_{z}^{w}\left\{\chi(\delta / \lambda, x / \lambda, z) G(\lambda z) \exp \left[\lambda^{-2}(F(\lambda z)-b)\right]\right\}
$$

and

$$
h_{2}(\lambda)=E_{z}^{w}\left\{[1-\chi(\delta / \lambda, x / \lambda, z)] G(\lambda z) \exp \left[\lambda^{-2}(F(\lambda z)-b)\right]\right\}
$$

From Lemma $9,\left|h_{2}(\lambda)\right| \leqq \gamma$ if $\lambda$ is sufficiently small. Choose $\lambda$ this small and consider $h_{1}(\lambda)$. From the translation theorem it follows that, letting $y=z-x / \lambda$,

$$
\begin{aligned}
h_{1}(\lambda)= & \exp \left(\frac{1}{2} \lambda^{-2} \int_{0}^{T}\left[x^{\prime}(\tau)\right]^{2} d \tau\right) \\
& \times E_{y}^{w}\left\{\chi(\delta / \lambda, 0, y) G(\lambda y+x) \exp \left[\lambda^{-1} \int_{0}^{T} x^{\prime}(\tau) d y(\tau)+\lambda^{-2}(F(\lambda y+x)-b)\right]\right\}
\end{aligned}
$$

Since $F(z)$ has two Frechet derivatives in a $\delta$ neighborhood of $x$, we may, by an extended form of Taylor's Theorem [7], write

$$
F(\lambda y+x)=f_{0}(0)+\lambda f_{1}(0) y+\lambda^{2} f_{2}(\eta) y^{2}
$$

where $0 \leqq\|\eta\| \leqq \delta$. Thus 


$$
\begin{aligned}
h_{1}(\lambda)= & \exp \left(\lambda^{-2}\left[-\frac{1}{2} \int_{0}^{T}\left[x^{\prime}(\tau)\right]^{2} d \tau+f_{0}(0)-b\right]\right) \\
& \times E_{y}^{w}\left\{\chi(\delta / \lambda, 0, y) G(\lambda y+x) \exp \left(\lambda^{-1}\left[f_{1}(0) y-\int_{0}^{T} x^{\prime}(\tau) d y(\tau)\right]+f_{2}(\eta) y^{2}\right)\right\}
\end{aligned}
$$

By hypothesis $F(z)-\frac{1}{2} \int_{0}^{T}\left[z^{\prime}(\tau)\right]^{2} d \tau$ has a maximum at $x$ over $C^{*}[0, T]$; therefore, its first variation vanishes over $C^{*}[0, T]$. Thus

$$
\begin{aligned}
0 & =\left.\frac{\partial}{\partial \varepsilon}\left(F(x+\varepsilon \eta)-\frac{1}{2} \int_{0}^{T}\left[x^{\prime}(\tau)+\varepsilon \eta^{\prime}(\tau)\right]^{2} d \tau\right)\right|_{\varepsilon>0} \\
& =f_{1}(0) \eta-\int_{0}^{T} x^{\prime}(\tau) d \eta(\tau)
\end{aligned}
$$

if $\eta \in C^{*}[0, T]$. By Lemma 10 ,

$$
f_{1}(0) \eta-\int_{0}^{T} x^{\prime}(\tau) d \eta(\tau)=0
$$

for $\eta \in C[0, T]$.

Since

$$
b=F(x)-\frac{1}{2} \int_{0}^{T}\left[x^{\prime}(\tau)\right]^{2} d \tau
$$

it follows that

$$
h_{1}(\lambda)=E_{y}^{w}\left\{\chi(\delta / \lambda, 0, y) G(\lambda y+x) \exp \left(f_{2}(\eta) y^{2}\right)\right\}
$$

We will now show that

$$
\left|h_{1}(\lambda)-G(x) E_{z}^{w}\left\{\exp \left(f_{2}(0) z^{2}\right)\right\}\right| \leqq 3 \gamma
$$

Indeed,

$$
\begin{aligned}
\left|h_{1}(\lambda)-G(x) E_{z}^{w}\left\{\exp \left(f_{2}(0) z^{2}\right)\right\}\right| \leqq & E_{y}^{w}\left\{\chi(\delta / \lambda, 0, y)|G(\lambda y+x)-G(x)| \exp \left(f_{2}(\eta) y^{2}\right)\right\} \\
& +E_{y}^{w}\left\{1-\chi(\delta / \lambda, 0, y)|G(x)| \exp \left(f_{2}(\eta) y^{2}\right)\right\} \\
& +|G(x)| E_{y}^{w}\left\{\left|\exp \left(f_{2}(\eta) y^{2}\right)-\exp \left(f_{2}(0) y^{2}\right)\right|\right\}
\end{aligned}
$$

and by choice of $\delta$ and for small enough $\lambda$ the last expression is less than or equal to $3 \gamma$. This proves Theorem $B$.

Proof of Theorem C. Choose $\delta$ so that assumptions 1, 2, and 3 of this Theorem hold. It can be shown [7] that the hypothesis on $F(z)$ implies

$$
F(\lambda z+x)=f_{0}(0)+\lambda f_{1}(0) z+\cdots+\lambda^{j-1} f_{j-1} z^{j-1}+k_{j}(\lambda z)
$$


where $k_{j}(\lambda z)=O\left(\lambda^{\jmath}\right), j=1, \ldots, n$ if $\|z\| \leqq \delta$. As in the proof of Theorem B, we may show that

$$
E_{z}^{w}\left\{G(\lambda z) \exp \left(\lambda^{-2} F(\lambda z)\right)\right\}=h_{1}(x)+h_{2}(\lambda)
$$

where $h_{2}(x)=0\left(\exp \left(\alpha \lambda^{-2}\right)\right)$ where $\alpha<0$ and

$$
h_{1}(\lambda)=E_{y}^{w}\left\{\chi(\delta / \lambda, 0, y) G(\lambda y+x) \exp \left[f_{2}(0) y^{2}+\lambda^{-2} k_{3}(\lambda y)\right]\right\} .
$$

It can be seen, using the Taylor expansion of $e^{x}$, that

$$
e^{x}=\sum_{i=0}^{n-1} x^{i} / i !+R_{n}(x)
$$

where

$$
\begin{array}{ll}
\left|R_{n}(x)\right| \leqq\left(x^{n} / n !\right) e^{x} & \text { if } x \geqq 0, \\
\left|R_{n}(x)\right| \leqq|x|^{n} / n ! & \text { if } x<0 .
\end{array}
$$

We may now write

$$
h_{1}(\lambda)=\sum_{i=0}^{n-3}(1 / i !) E_{y}^{w}\left\{\chi(\delta / \lambda, 0, y) G(\lambda y+x) \exp \left(f_{2}(0) y^{2}\right)\left[\lambda^{-2} k_{3}(\lambda y)\right]^{i}\right\}+J_{n-2}(\lambda) .
$$

Let $B(\lambda, y)$ be the characteristic function of the set $\left\{y \in C[0, T] \mid k_{3}(\lambda y) \geqq 0\right\}$. It can be seen that

$$
\begin{array}{r}
J_{n-2}(\lambda) \leqq(1 /(n-2) !) E_{y}^{w}\left\{\chi(\delta / \lambda, 0, y)|G(\lambda y+x)| \cdot\left|\lambda^{-2} k_{3}(\lambda y)\right|^{n-2}\right. \\
\left.\cdot \exp \left(f_{2}(0) y^{2}+\lambda^{-2} k_{3}(\lambda y)\right) B(\lambda, y)\right\} \\
+(1 /(n-2) !) E_{y}^{w}\left\{\chi(\delta / \lambda, 0, y)|G(\lambda y+x)| \cdot\left|\lambda^{-2} k_{3}(\lambda y)\right|^{n-2}\right. \\
\left.\cdot \exp \left(f_{2}(0) y^{2}\right)[1-B(\lambda, y)]\right\} .
\end{array}
$$

From Taylor's Theorem for functionals it follows that if $\|\lambda y\| \leqq \delta$ then

$$
\lambda^{2} f_{2}(0) y^{2}+k_{3}(\lambda y)=k_{2}(\lambda y)=\lambda^{2} f_{2}(\eta) y^{2}
$$

where $\|\eta\| \leqq \delta$. By hypothesis $\left[k_{3}(\lambda y)\right] \leqq C_{3} \lambda^{3}\|y\|^{3}$, where $0 \leqq C_{3}<\infty$. Thus

$$
\begin{aligned}
&\left|J_{n-2}(\lambda)\right| \leqq(1 /(n-2) !) E_{y}^{w}\left\{\chi(\delta / \lambda, 0, y)|G(\lambda y+x)|\left(C_{3} \lambda\right)^{n-2}\|y\|^{3(n-2)}\right. \\
&\left.\cdot \exp \left(f_{2}(\eta) y^{2}\right) B(\lambda, y)\right\} \\
&+(1 /(n-2) !) E_{y}^{w}\left\{\chi(\delta / \lambda, 0, y)|G(\lambda y+x)|\left(C_{3} \lambda\right)^{n-2}\|y\|^{3(n-2)}\right. \\
&\left.\cdot \exp \left(f_{2}(0) y^{2}\right)[1-B(\lambda, y)]\right\} .
\end{aligned}
$$

From the Hölder inequality, and assumption 2 of this theorem, it follows that $J_{n-2}(\lambda)=O\left(\lambda^{n-2}\right)$. 
Thus we have obtained

$$
h_{1}(\lambda)=\sum_{i=0}^{n-3}(1 / i !) E_{y}^{w}\left\{\chi(\delta / \lambda, 0 ; y) G(\lambda y+x) \exp \left(f_{2}(0) y^{2}\right)\left[\lambda^{-2} k_{3}(\lambda y)\right]^{i}\right\}+O\left(\lambda^{n-2}\right) .
$$

Since $G(z)$ has $n-2$ Frechet derivatives in a neighborhood of $x$, we may write

$$
G(\lambda y+x)=\sum_{j=0}^{n-3} \lambda^{j} g_{j} y^{j}+L_{n-2}(\lambda y)
$$

where

$$
L_{n-2}(\lambda y)=O\left(\|\lambda y\|^{n-2}\right)
$$

Thus

$$
h_{1}(\lambda)=\sum_{i=0}^{n-3} \sum_{j=0}^{n-3}(1 / i !) \lambda^{j} E_{y}^{w}\left\{\chi(\delta / \lambda, 0, y) g_{j} y^{j} \exp \left(f_{2}(0) y^{2}\right)\left[\lambda^{-2} k_{3}(\lambda y)\right]^{i}\right\}+O\left(\lambda^{n-2}\right)
$$

since

$$
\sum_{i=0}^{n-3}(1 / i !) E_{y}^{w}\left\{\chi(\delta / \lambda, 0, y) O\left(\|\lambda y\|^{n-2}\right) \exp \left(f_{2}(0) y^{2}\right)\left[\lambda^{-2} k_{3}(\lambda y)\right]^{i}\right\}=O\left(\lambda^{n-2}\right) .
$$

It can be seen from assumption 2, the Hölder inequality, Lemma 3 and the fact that $k_{3}(\lambda y)=C_{3} \lambda^{3}\|y\|^{3}$ that

$$
\sum_{i=0}^{n-3} \sum_{j=0}^{n-3}(1 / i !) \lambda^{j} E_{y}^{w}\left\{[1-\chi(\delta / \lambda, 0, y)] g_{j} y^{j} \exp \left(f_{2}(0) y^{2}\right)\left[\lambda^{-2} k_{3}(\lambda y)\right]^{i}\right\}=O\left(\lambda^{n-2}\right) .
$$

We may now write

$$
h_{1}(\lambda)=\sum_{i=0}^{n-3} \sum_{j=0}^{n-3}(1 / i !) \lambda^{j} E_{y}^{w}\left\{g_{j} y^{j} \exp \left(f_{2}(0) y^{2}\right)\left[\lambda^{-2} k_{3}(\lambda y)\right]^{j}\right\}+O\left(\lambda^{n-2}\right),
$$

since

$$
\begin{aligned}
k_{3}(\lambda y)= & \lambda^{3} f_{3}(0) y^{3}+\cdots+\lambda^{n-1} f_{n-1}(0) y^{n-1}+k_{n}(\lambda y), \\
h_{1}(\lambda)= & \sum_{i=0}^{n-3} \sum_{j=0}^{n-3}(1 / i !) \lambda^{j} E_{y}^{w}\left\{g_{j} y^{j} \exp \left(f_{2}(0) y^{2}\right)\right. \\
& \left.\quad \times\left[\lambda f_{3}(0) y^{3}+\cdots+\lambda^{n-3} f_{n-1}(0) y^{n-1}+\lambda^{-2} k_{n}(\lambda y)\right]^{j}\right\}+O\left(\lambda^{n-2}\right) .
\end{aligned}
$$

Expanding out we find that

$$
\begin{aligned}
h_{1}(\lambda)=\sum_{i=0}^{n-3} \sum_{j=0}^{n-3}\left(\lambda^{j} / i !\right) E_{y}^{w} & \left\{g_{j} y^{j} \exp \left(f_{2}(0) y^{2}\right)\right. \\
& \left.\times\left[\lambda f_{3}(0) y^{3}+\cdots+\lambda^{n-3} f_{n-1}(0) y^{n-1}\right]^{i}\right\}+O\left(\lambda^{n-2}\right)
\end{aligned}
$$

since

$$
k_{n}(\lambda y) / \lambda^{2}=O\left(\lambda^{n-2}\right)
$$


Clearly we may rewrite $h_{1}(\lambda)$ as

$$
h_{1}(\lambda)=\Gamma_{0} \lambda+\Gamma_{1} \lambda+\cdots+\Gamma_{n-3} \lambda^{n-3}+O\left(\lambda^{n-2}\right)
$$

which proves Theorem $\mathrm{C}$.

5. Applications of Theorem A. In this section some applications of Theorem A will be given. First an algorithm for solving certain types of functional equations will be developed, and then this algorithm will be used to solve a problem in differential equations. Also, a problem in the calculus of variations will be solved.

It is easy to see that if the functional $G(z)$ is $z$ evaluated at the point $s$, i.e., $G(z)=z(s)$, then $G(z)$ satisfies conditions 1,2 , and 4 of $\$ 2$. It follows from Theorem A that if $F(z)$ satisfied conditions 1,3 , and 5 of $\S 2$, and $F(z)-\frac{1}{2} \int_{0}^{T}\left[z^{\prime}(\tau)\right]^{2} d \tau$ has a proper maximum over $C^{*}[0, T]$ at $x$, then

$$
x(s)=\lim _{\lambda \rightarrow 0} \frac{E_{z}^{w}\left\{\lambda z(s) \exp \left(\lambda^{-2} F(\lambda z)\right)\right\}}{E_{z}^{w}\left\{\exp \left(\lambda^{-2} F(\lambda z)\right)\right\}}
$$

i.e., we have a formula for the function which maximizes

$$
F(z)-\frac{1}{2} \int_{0}^{T}\left[z^{\prime}(\tau)\right]^{2} d \tau
$$

over $C^{*}[0, T]$.

Suppose the functional equation $H(x)=0$ has a solution $x \in C^{*}[0, T]$, and, moreover, suppose $H(x)$ is such that there exists a functional $F(z)$ for which

$$
F(z)-\frac{1}{2} \int_{0}^{T}\left[z^{\prime}(\tau)\right]^{2} d \tau
$$

has a proper maximum over $C^{*}[0, T]$ at the same point $x$. Then assuming $F(z)$ satisfies the hypothesis of Theorem A, (5.1) becomes a formula for the solution of the functional equation $H(x)=0$.

As an example of this algorithm, the following theorem will be proved.

THEOREM D. Suppose $f(x)$ is Lipschitz continuous on bounded sets, real valued, and defined on $-\infty<x<\infty$, and that $|f(x)| \leqq c x$ where $c$ is a constant and $c<1 / 2 T$. Then the solution to the differential equation

$$
\phi^{\prime}(s)=f(\phi(s))
$$

with $\phi(0)=0$ is given by

$$
\phi(s)=\lim _{\lambda \rightarrow 0} \frac{E_{z}^{w}\left\{\lambda z(s) \exp \left[2 \lambda^{-2} \int_{0}^{T} f^{2}(\lambda z(\tau)) d \tau+\lambda^{-2} \int_{0}^{\lambda z(T)} f(u) d u\right]\right\}}{E_{z}^{w}\left\{\exp \left[2 \lambda^{-2} \int_{0}^{T} f^{2}(\lambda z(\tau)) d \tau+\lambda^{-2} \int_{0}^{\lambda z(T)} f(u) d u\right]\right\}} .
$$


Proof. From the above reasoning it is sufficient to show that

$$
-\frac{1}{2} \int_{0}^{T} f^{2}(z(\tau)) d \tau+\int_{0}^{z(T)} f(u) d u-\frac{1}{2} \int_{0}^{T}\left[z^{\prime}(\tau)\right]^{2} d \tau
$$

has a proper maximum over $C^{*}[0, T]$ at $\phi$, that $\phi$ solves the differential equation (5.2), and that

$$
F(z)=-\frac{1}{2} \int_{0}^{T} f^{2}(z(\tau)) d \tau+\int_{0}^{z(T)} f(u) d u
$$

satisfies conditions 1,3 , and 5 of Theorem A.

It is clear from hypothesis and Picard's theorem [2] that the functional

$$
H(z)=-\frac{1}{2} \int_{0}^{T}\left[f(z(\tau))-z^{\prime}(\tau)\right]^{2} d \tau, \quad z \in C^{*}[0, T],
$$

has a proper maximum at $\phi$, the solution to the differential equation (5.2). Clearly, also,

$$
\begin{aligned}
-\frac{1}{2} \int_{0}^{T}[f(z(s)) & \left.-z^{\prime}(s)\right]^{2} d s \\
& =-\frac{1}{2} \int_{0}^{T} f^{2}(z(s)) d s+\int_{0}^{z(T)} f(u) d u-\frac{1}{2} \int_{0}^{T}\left[z^{\prime}(s)\right]^{2} d s .
\end{aligned}
$$

It will now be shown that $F(z)$ (see (5.4)) satisfies conditions 1,3 , and 5 of $\S 2$. It can be seen that

$$
F(z)=-\frac{1}{2} \int_{0}^{T} f^{2}(z(s)) d s+\int_{0}^{z(T)} f(s) d s \leqq \int_{0}^{z(T)} f(s) d s \leqq(c / 2)\|z\|^{2} .
$$

It thus follows that condition 3 of $\$ 2$ holds. To show that conditions 1 and 5 of $\S 2$ hold, it is sufficient to show that $F(z)$ is uniformly continuous in the sup norm on bounded sets. This is easily shown.

The problem of finding the maximum of

$$
\int_{0}^{T}\left[p(z(\tau), \tau)-\frac{1}{2}\left[z^{\prime}(\tau)\right]^{2}\right] d \tau+\phi(z(T))
$$

will now be considered.

It follows from the reasoning given at the beginning of this section that if the functional $F(z)=\int_{0}^{T} p(z(\tau), \tau) d \tau+\phi(z(T))$ satisfies conditions 1,3 , and 5 of $\S 2$ and if $F(z)-\int_{0}^{T}\left[z^{\prime}(\tau)\right]^{2} d \tau$ has a proper maximum over $C^{*}[0, T]$ at $y$, then

$$
y(s)=\lim _{\lambda \rightarrow 0} \frac{E_{z}^{w}\left\{\lambda z(s) \exp \left(\lambda^{-2}\left[\int_{0}^{T} p(\lambda z(\tau), \tau) d \tau+\phi(\lambda z(T))\right]\right)\right\}}{E_{z}^{w}\left\{\exp \left(\lambda^{-2}\left[\int_{0}^{T} p(\lambda z(\tau), \tau) d \tau+\phi(\lambda z(T))\right]\right)\right\}} .
$$

The following theorem will be proved. 
Theorem E. Suppose $p(x, \tau)$ and $\phi(x)$ are two real valued continuous functions defined on $(-\infty<x<\infty),(0 \leqq \tau \leqq T)$ and $(-\infty<x<\infty)$ respectively, such that

1. $p(x, \tau) \leqq C_{1}+C_{2} x^{2}$ uniformly in $\tau$, and $\phi(x) \leqq C_{3}+C_{4} x^{2} . C_{1}$ and $C_{3}$ are any positive numbers but $C_{2} T+C_{4}<1 / 4 T$.

2. $p(x, \tau)$ is continuous in $x$ and $\tau$ and $\phi^{\prime}(x)$ is continuous in $x$.

3. $\partial[p(x, \tau)] / \partial x$ is continuous in $\tau$ and $x$.

4. The functional $\int_{0}^{T} p(z(\tau), \tau) d \tau-\frac{1}{2} \int_{0}^{T}\left[z^{\prime}(\tau)\right]^{2} d \tau+\phi(z(T))$ has a proper maximum on the class $C^{*}[0, T]$ at the function $y$.

Under conditions 1-4 it is true that the function $y(s)$ defined by (5.9) maximizes (5.8) and that the function $y(s)$ satisfies the following differential equation.

$$
p_{x}(y(\tau), \tau)+y^{\prime \prime}(\tau)=0
$$

with boundary conditions $y(0)=0$ and $y^{\prime}(T)=\phi^{\prime}(y(T))$.

Proof. Let $F(z)=\int_{0}^{T} p(z(\tau), \tau) d \tau+\phi(z(T))$. It can be seen that $F(z)$ is uniformly continuous in the sup norm on bounded sets.

It will be shown now that

$$
F(z) \leqq T C_{1}+C_{3}+\left(T C_{2}+C_{4}\right)\|z\|^{2}
$$

where $T C_{2}+C_{4}<1 / 4 T$. From the definition of $F(z)$ and assumption 1 of this theorem it follows that

$$
F(z) \leqq \int_{0}^{T}\left[C_{1}+C_{2} z^{2}(\tau)\right] d \tau+C_{3}+C_{4} z^{2}(\tau) \leqq T C_{1}+C_{3}+\left(T C_{2}+C_{4}\right)\|z\|^{2}
$$

Thus $F(z)$ of the present theorem satisfies conditions 1,2 , and 5 of $\S 2$, has a proper maximum over $C^{*}[0, T]$, and thus the maximizing function $y(s)$ of $(5.8)$ is given by (5.9).

From the calculus of variations theory it also follows that $y(s)$ satisfies an Euler equation which, in the case considered, is $p_{x}(y(\tau), \tau)+y^{\prime \prime}(\tau)=0$ with the boundary conditions $y(0)=0$ and $y^{\prime}(T)=\phi^{\prime}(y(T))$ [3]. This proves Theorem E.

Suppose $F(z)$ satisfies the hypothesis of Theorem A and that the functional $F(z)-\frac{1}{2} \int_{0}^{T}\left[z^{\prime}(\tau)\right]^{2} d \tau$ has a proper maximum on $C^{*}[0, T]$ and as usual let us denote the maximizing function by $x(\tau)$. We note that

$$
x(s)=\lim _{\lambda \rightarrow 0} \frac{E_{z}^{w}\left\{\lambda z(s) \exp \left(\lambda^{-2} F(\lambda z)\right)\right\}}{E_{z}^{w}\left\{\exp \left(\lambda^{-2} F(\lambda z)\right)\right\}}
$$

gives us a formula for a maximizing function even if the functional $F(z)$ does not have a Frechet derivative. Thus formula $(5.10)$ is an expression for the maximizing function even if there is no Euler equation for the calculus of variations problem. 


\section{REFERENCES}

1. R. H. Cameron and W. T. Martin, Transformations of Wiener integrals under translations, Ann. of Math. 45 1955, 386-396.

2. E. A. Coddington and N. Levinson, Theory of ordinary differential equations, McGrawHill, New York, 1955, p. 10.

3. R. Courant and D. Hilbert, Methods of mathematical physics, Vol. 1, Interscience, New York, 1953, p. 210.

4. J. L. Doob, Stochastic processes, Wiley, New York, 1953.

5. A. Erdélyí, Asymptotic expansions, Dover, New York, 1956, pp. 36-39.

6. P. Erdös and M. Kac, On certain limit theorems of the theory of probability, Bull. Amer. Math. Soc. 52 (1946), 292-302.

7. V. Volterra, Theory of functionals and of integral and integro-differential equations, Dover, New York, 1959, pp. 25-26.

8. N. Wiener, Generalized harmonic analysis, Acta Math. 55 (1930), 117-258.

\section{ibM Watson Research Center,}

YorkTOWN HeIGHTS, NEW YoRK 